\title{
Representation of vector fields
}

\author{
A. G. Ramm \\ Mathematics Department, Kansas State University, Manhattan, KS, 66502, USA \\ Email: ramm@math.ksu.edu
}

Copyright (C)2015 A. G. Ramm. This is an open access article distributed under the Creative Commons Attribution License, which permits unrestricted use, distribution, and reproduction in any medium, provided the original work is properly cited.

\begin{abstract}
A simple proof is given for the explicit formula which allows one to recover a $C^{2}-\operatorname{smooth}$ vector field $A=A(x)$ in $\mathbb{R}^{3}$, decaying at infinity, from the knowledge of its $\nabla \times A$ and $\nabla \cdot A$. The representation of $A$ as a sum of the gradient field and a divergence-free vector fields is derived from this formula. Similar results are obtained for a vector field in a bounded $C^{2}$-smooth domain.
\end{abstract}

Keywords: vector fields; representation of vector fields.

\section{Introduction}

In fluid mechanics and electrodynamics one is often interested in the following questions:

Q1. Let $A(x), x \in \mathbb{R}^{3}$, be a twice differentiable in $\mathbb{R}^{3}$ vector field vanishing at infinity together with its two derivatives. Given $\nabla \times A$ and $\nabla \cdot A$, can one recover $A(x)$ uniquely? Can one give an explicit formula for $A(x)$ ?

Q2. Can one find a scalar field $u=u(x)$ and a divergence-free vector field $B(x), \nabla \cdot B=0$, such that

$$
A=\nabla u+B, \quad \int_{\mathbb{R}^{3}} \nabla u \cdot B d x=0 .
$$

These questions were widely discussed in the literature, for example, in [1] - [3]. Our aim is to give a simple answer to these questions. By $H^{m}\left(\mathbb{R}^{3}\right), H^{m}(D)$, the usual Sobolev spaces are denoted, $H^{m}(D, w(x))$ is the weighted Sobolev space, where $w=w(x)>0$ is the weight function.

\section{Answer to question Q1.}

Denote $\nabla \times A:=a, \nabla \cdot A:=f$. Then $\nabla \times \nabla \times A=\nabla \times a$. It is well known that

$-\nabla^{2} A=\nabla \times \nabla \times A-\nabla \nabla \cdot A$.

Thus,

$-\nabla^{2} A=\nabla \times a-\nabla f$ 
Let $g(x, y):=\frac{1}{4 \pi|x-y|}$. Then

$-\Delta g(x, y)=\delta(x-y)$

where $\delta(x)$ is the delta function. Thus, from (3) one gets

$A(x)=\int_{\mathbb{R}^{3}} g(x, y) \nabla \times a d y-\int_{\mathbb{R}^{3}} g(x, y) \nabla f d y$.

This formula gives an analytical representation of $A(x)$ in terms of $a=\nabla \times A$ and $f=\nabla \cdot A$.

To prove the uniqueness of this representation, assume that there are two different vector fields $A$ and $F$ that have the same $a=\nabla \times A=\nabla \times F$ and $f=\nabla \cdot A=\nabla \cdot F$. Then, by formula (2), one has

$-\nabla^{2}(A-F)=0$.

Therefore $A-F$ is a harmonic function in $\mathbb{R}^{3}$ which vanishes at infinity. By the maximum principle such a function is equal to zero identically.

Thus, $A(x)$ is uniquely determined in $\mathbb{R}^{3}$ by formula (5) if $\nabla \times A$ and $\nabla \cdot A$ are known and if $A$ vanishes at infinity.

\section{Answer to question Q2.}

Formula (5) can be written as

$A(x)=\nabla \times \int_{\mathbb{R}^{3}} g(x, y) a(y) d y-\nabla \int_{\mathbb{R}^{3}} g(x, y) f(y) d y$,

provided that $a(y)$ and $f(y)$ decay at infinity sufficiently fast, for example, if

$$
|A(x)|+|\partial A(x)|+\left|\partial^{2} A(x)\right| \leq c(1+|x|)^{-\gamma}, \gamma>3,
$$

where $\partial$ is an arbitrary first order derivative, so that the following integrations by parts can be justified:

$$
\begin{aligned}
& \nabla \times \int_{\mathbb{R}^{3}} g(x, y) a(y) d y=-\int_{\mathbb{R}^{3}}\left[\nabla_{y} g(x, y), a(y)\right] d y=\int_{\mathbb{R}^{3}} g(x, y) \nabla \times a(y) d y, \\
& -\nabla \int_{\mathbb{R}^{3}} g(x, y) f(y) d y=\int_{\mathbb{R}^{3}} \nabla_{y} g(x, y) f(y) d y=-\int_{\mathbb{R}^{3}} g(x, y) \nabla f(y) d y .
\end{aligned}
$$

One may also assume that $A \in H^{2}\left(\mathbb{R}^{3}, 1+|x|^{\gamma}\right), \gamma>2$, in order to justify formulas (1), (5), (7).

It follows from (1) and (7) that

$u(x)=-\int_{\mathbb{R}^{3}} g(x, y) f(y) d y, \quad B(x)=\nabla \times \int_{\mathbb{R}^{3}} g(x, y) a(y) d y$.

To check the second formula (1), it is sufficient to check that

$\int_{\mathbb{R}^{3}} \nabla u \cdot \nabla \times p d x=0$,

provided that $u=u(x)$ and $p=p(x)$ decay at infinity sufficiently fast. In our case $u$ is defined by formula (10) and $p(x)=\int_{\mathbb{R}^{3}} g(x, y) a(y) d y$.

Formula (11) can be verified by a direct calculation. Let $\frac{\partial u}{\partial x_{j}}:=u_{, j}$ and denote by $e_{j m q}$ the antisymmetric unit tensor: $e_{123}=1, e_{j m q}=\left\{\begin{array}{ll}1 & \text { if } j m q \text { is even, } \\ -1 & \text { if } j m q \text { is odd. }\end{array}\right.$ The triple $j m q$ is called even if by an even number of transpositions it can be reduced to the triple 123. An odd triple $j m q$ is the one that is not even. A transposition is the change of the order of two neighboring indices.

The vector product can be written with the help of $e_{j m q}$ as follows:

$$
(A \times B)_{j}=e_{j m q} A_{m} B_{q} .
$$


Here and below summation is understood over the repeated indices. For example, $(\nabla \times p)_{j}=e_{j m q} p_{q, m}$, where $p_{q, m}:=\frac{\partial p_{q}}{\partial x_{m}}$. With these notations one has

$\int_{\mathbb{R}^{3}} \nabla u \cdot \nabla \times p d x=e_{j m q} \int_{\mathbb{R}^{3}} u, j p_{q, m} d x=-e_{j m q} \int_{\mathbb{R}^{3}} u p_{q, m j} d x=0$,

because $e_{j m q} p_{q, m j}=0$.

Let us summarize the results.

Theorem 3.1 Assume that a vector field $A(x) \in H_{l o c}^{2}\left(\mathbb{R}^{3}\right)$ decays at infinity sufficiently fast, for example, A $(x) \in$ $H^{2}\left(\mathbb{R}^{3}, 1+|x|^{\gamma}\right), \gamma>2$. Then, given $a:=\nabla \times A$ and $f:=\nabla \cdot A$ in $\mathbb{R}^{3}$ one can uniquely recover $A$ by formula $(5)$.

Moreover, one can uniquely represent $A(x)$ by formula (1), where $u$ and $B$ are uniquely defined by formula (10).

Theorem 3.2 Assume that $D \subset \mathbb{R}^{3}$ is a bounded domain with $C^{2}$-smooth boundary $S, A(x) \in H^{2}(D)$, a $(x):=$ $\nabla \times A(x), f:=\nabla \cdot A(x)$ and $\phi(s)=\left.A\right|_{s \in S}$ are known. Then $A(x)$ is uniquely recovered by solving the Dirichlet problem

$-\nabla^{2} A=\nabla \times a(x)-\nabla f(x)$ in $D,\left.\quad A\right|_{S}=\phi(s)$.

Proof. Theorem 3.1 is already proved.

To prove Theorem 3.2 one reduces it to solving problem (13). Existence and uniqueness of the solution to the Dirichlet problem (13) are known, so Theorem 3.2 is proved.

Remark 3.1 It follows from formula (7) that if $f=\nabla \cdot A=0$, then $A=B=\nabla \times \int_{\mathbb{R}^{3}} g(x, y) a(y) d y$, and if $a=\nabla \times A=0$, then $A=\nabla u$, where $u$ is defined in formula (10).

Remark 3.2 Under the assumption $\gamma>3$, vector field $A(x)$ decays at infinity so that formulas (1), (5), and (10) are valid.

Let us estimate, for example, an integral of the type (9) assuming that $|\nabla f| \leq \frac{c}{(1+|x|)^{\gamma}}, \gamma>3$. Let $|x|=r,|y|=\rho$, $\theta$ be the angle between $x$ and $y$, and $x$ is directed along $y_{3}$ axis. Then one has

$$
\begin{aligned}
I_{1}: & =\int_{\mathbb{R}^{3}} \frac{d y}{|x-y|(1+|y|)^{\gamma}} \\
& =2 \pi \int_{0}^{\infty} \frac{d r r^{2}}{(1+r)^{\gamma}} \int_{-1}^{1} \frac{d s}{\left(r^{2}-2 r \rho s+\rho^{2}\right)^{1 / 2}} \\
& =\frac{\pi}{\rho} \int_{0}^{\infty} \frac{d r r}{(1+r)^{\gamma}}(r+\rho-|r-\rho|) \\
& =\frac{\pi}{\rho}\left(2 \int_{0}^{\rho} \frac{d r r^{2}}{(1+r)^{\gamma}}+\int_{\rho}^{\infty} \frac{d r r 2 \rho}{(1+r)^{\gamma}}\right) \\
& \leq \frac{\pi}{\rho}\left(\left.2 \frac{(1+r)^{-\gamma+3}}{-\gamma+3}\right|_{0} ^{\rho}+\left.2 \rho \frac{(1+r)^{-\gamma+2}}{-\gamma+2}\right|_{\rho} ^{\infty}\right) \\
& \leq \frac{2 \pi}{\rho(\gamma-3)}+\frac{2 \pi}{\gamma-2} \frac{1}{\rho^{\gamma-2}} .
\end{aligned}
$$

If $A \in H^{2}\left(\mathbb{R}^{3},(1+|x|)^{\gamma}\right), \gamma>2$, let us estimate, for example, the following integral:

$$
\begin{aligned}
I_{2}^{2} & :=\left(\int_{\mathbb{R}^{3}} \frac{1}{|x-y|}|\nabla \times a| d y\right)^{2} \\
& \leq \int_{\mathbb{R}^{3}} \frac{d y}{|x-y|^{2}(1+|y|)^{\gamma}} \int_{\mathbb{R}^{3}}|\nabla \times a|^{2}(1+|y|)^{\gamma} d y \\
& \leq c \int_{\mathbb{R}^{3}} \frac{d y}{|x-y|^{2}(1+|y|)^{\gamma}} \\
& \leq 2 \pi c \int_{0}^{\infty} \frac{d r r^{2}}{(1+r)^{\gamma}} \int_{-1}^{1} \frac{d s}{r^{2}-2 r \rho s+\rho^{2}} \\
& =\frac{\pi c}{\rho} \int_{0}^{\infty} \frac{d r r}{(1+r)^{\gamma}} \ln \frac{c_{1} \ln \rho}{\rho}, \quad \rho>1 .
\end{aligned}
$$

By $c, c_{1}>0$ estimation constants are denoted. 


\section{References}

[1] O. Ladyzhenskaya, The mathematical theory of viscous incompressible fluid, Gordon and Breach, New York, 1969.

[2] D. Menzel, Fundamental formulas of physics, Prentice Hall, New York, 1955.

[3] R. Temam, Navier-Stokes equations, North Holland, Amsterdam, 1984. 